\title{
Who knew? Awareness of being recommended for influenza vaccination among US adults
}

\author{
Jürgen Maurer, ${ }^{a}$ Katherine M. Harris, ${ }^{b}$ Andrew M. Parker ${ }^{c}$ \\ ${ }^{\mathrm{a}}$ Institute of Health Economics and Management, University of Lausanne and RAND Corporation, Arlington, VA, USA. ${ }^{\mathrm{B}}$ RAND Corporation, \\ Arlington, VA, USA. ${ }^{\mathrm{R}}$ RAND Corporation, Pittsburgh, PA, USA. \\ Correspondence: Jürgen Maurer, IEMS, University of Lausanne, Route de Chavannes 31, 1015 Lausanne, Switzerland. E-mail: jurgen.maurer@unil.ch
}

Accepted 9 October 2011. Published Online 25 November 2011.

Background Starting with the 2010-2011 influenza season, the Advisory Committee on Immunization Practices at the US Centers for Disease Control and Prevention recommends annual influenza vaccination to all people aged 6 months and older unless contraindicated.

Objectives To measure perceived influenza vaccination recommendation status among US adults $(n=2122)$ and its association with socio-demographic characteristics and recommendation status during the 2009-2010 pandemic influenza season.

Methods We analyze nationally representative data from longitudinal Internet surveys of US adults conducted in November-December 2009 and September-October 2010.

Results During the 2010-2011 vaccination season, $46 \cdot 2$ percent (95\%-CI: $43 \cdot 3-49 \cdot 1 \%$ ) of US adults correctly reported to be covered by a government recommendation for influenza vaccination. Awareness of being covered by a government influenza vaccination recommendation was statistically significantly higher among non-working adults and adults who had been recommended for seasonal vaccination or both seasonal and H1N1 vaccination during the 2009-2010 pandemic influenza vaccination season.

Conclusion Our results highlight that a majority of US adults do not know that they are recommended for annual influenza vaccination by the government. The fraction of adults who are unaware of their recommendation status is especially large among newly recommended healthy young adults. The universal vaccination recommendations will only be successful if they reach both patients and physicians and lead to changing vaccination practices. The universal nature of the new recommendation simplifies vaccination-related outreach and compliance with government vaccination guidelines considerably, as it does not require any identification of specific recommendation groups based on complex personal or health risk factors.

Keywords ACIP recommendations, influenza, influenza vaccination, patient knowledge.

Please cite this paper as: Maurer et al. (2012) Who knew? Awareness of being recommended for influenza vaccination among US adults. Influenza and Other Respiratory Viruses 6(4), 284-290.

\section{Introduction}

The Advisory Committee on Immunization Practices (ACIP) at the US Centers for Disease Control and Prevention $(\mathrm{CDC})^{1}$ now recommends annual influenza vaccination for all people aged 6 months and older unless contraindicated. The ACIP is the only entity in the US federal government that formulates recommendations on the use of vaccines by the US civilian population based on careful review of the scientific evidence. ${ }^{2}$ Through endorsements by medical professional societies and public health authorities, ACIP recommendations influence the nature of public health communication about vaccination and the vaccine-related practices of healthcare provides. The new
ACIP recommendations of near universal annual influenza vaccination were also publicized in the news media and on the Internet, using some of the same communication tools that had been deployed for informing the public during the 2009 influenza A/H1N1 pandemic (see, e.g., Flu.gov, Accessed 9 August 2011). ${ }^{3}$

Prior to the 2010-2011 vaccination season, annual vaccination for influenza was based on a complex array of personal characteristics such as age, occupation, living arrangements, and existing health conditions that put individuals at elevated risk of transmitting and contracting influenza or suffering serious complications from a potential influenza infection. ${ }^{4}$ Pieced together, the risk-based recommendations covered roughly $80 \%$ of the total US population. 
By extending recommendations to all healthy adults aged 18-49 years, the ACIP influenza vaccination recommendations became near universal. While the merits of a near universal influenza vaccination recommendation have long been debated, the recent pandemic brought home the fact that novel strains of influenza can cause severe illness in otherwise healthy, "low-risk" people. Moreover, vaccinating healthy people also helps to protect their close contacts who may be highly vulnerable to influenza and its complications.

The new universal recommendation will also simplify administrative procedures for influenza vaccination according to ACIP guidelines by eliminating the step of verifying patients' recommendation status before discussing vaccination with patients in clinical practice. For example, previous research indicates that only one half of physician practices were able to generate lists of patients with chronic illnesses at high risk for complications of influenza. These practices, in turn, were significantly more likely to use patient reminders for influenza vaccination. ${ }^{5}$

While the universal vaccination recommendation simplifies administrative procedures, it does not eliminate the need to identify subgroups with low vaccination rates, who are unaware of being recommended for annual influenza vaccination and to target information about the benefits of vaccination to these patient groups. To date, there is no evidence regarding whether the US public is aware of the new universal recommendation. Based on the experience of last year's pandemic, we suspect that healthy young adults remain relatively unaware of being newly recommended for annual influenza vaccination. During the pandemic, only one-third of US adults who were recommended for either or both seasonal or H1N1 influenza vaccination were aware of it, highlighting gaps in public awareness about the risks and benefits of influenza vaccination. ${ }^{6}$ To test this hypothesis, we analyzed recently collected nationally representative data on perceived recommendation status for influenza vaccination among US adults and estimated its association with socio-demographic characteristics and coverage by ACIP's previous, less comprehensive influenza vaccination recommendations for the 2009-2010 pandemic vaccination season. Comparing the perceived recommendation status of newly and previously recommended persons, thereby, allows us to assess whether the new universal recommendations have already resulted in levels of awareness among newly recommended adults that are comparable with those among persons who have already been recommended for influenza vaccination during past vaccination seasons.

\section{Methods}

We employed survey data from two waves (9 and 12) of a twelve-wave longitudinal survey effort to monitor US adults' knowledge, attitudes, beliefs, and behaviors with regard to influenza and influenza prevention during the 2009-2010 influenza pandemic. The surveys were administered to members of RAND's American Life Panel (ALP), a population-based Internet panel of US adults. The ALP covers households with and without Internet access at the time of recruitment by supplying WebTV or laptop computers and high-speed Internet to participants who do not have Internet access at the time of recruitment. A detailed description of the panel as well as the questionnaires and data used in this study is publicly available at https:// mmicdata.rand.org/alp/index.php/Main_Page.

The data for this analysis were collected between November 19, 2009 and December 10, 2009 (wave 9) and September 22, 2010 and October 25, 2010 (wave 12). Data on our outcome of interest - adults' knowledge of their own influenza vaccination recommendation status - were collected in wave 12 of the survey, which was completed by 2628 ALP members (completion rate: $80 \cdot 8$ percent). 80.7 percent of wave 12 respondents had previously completed wave 9 of the survey, which gathered comprehensive information about respondents' influenza risk characteristics corresponding to the criteria used by ACIP to define its seasonal and $\mathrm{H} 1 \mathrm{~N} 1$ vaccination recommendations during the 2009-2010 pandemic. ${ }^{4,7}$ Our final analytical sample consisted of $n=2122$ respondents who responded to both survey waves (waves 9 and 12). Combining the two survey waves did not only allow us to measure adults' awareness of being currently covered by an influenza vaccination recommendation, but also permitted us to study the relationship between their current awareness of their recommendation status for influenza vaccination relative to their recommendation status during the 2009 influenza $\mathrm{A} / \mathrm{H} 1 \mathrm{~N} 1$ pandemic.

Awareness of being recommended for influenza during the 2010-2011 vaccination season was measured by asking: "Each year, the federal government recommends which members of the general population should get a flu vaccine. According to government recommendations, are you recommended to get the flu vaccine this flu season (August 2010 to May 2011)? " Permissible answers were "Yes," "No," and "Don't know" and we combined the categories "No," and "Don't know," indicating lack of knowledge about being covered by a government vaccination recommendation for influenza.

We determined whether the respondents were recommended for seasonal and/or H1N1 vaccination during the 2009-2010 pandemic based on the risk factors measured in November/December 2009 (wave 9), the midpoint of the pandemic. During this time, both seasonal and pandemic vaccinations were recommended for healthcare workers, pregnant women, adults aged 25-64 years with a high-risk health condition, such as asthma, heart disease or diabetes, 
and adults with regular contact with children age 6 months or younger. Seasonal vaccination was recommended for all adults aged 50 years and older as well as for the persons in regular contacts with adults aged 50 years and older or children age 5 or younger. H1N1 vaccination was recommended for all adults aged 18-24 years. Based on corresponding measures available in survey wave 9 , we constructed detailed control variables for coverage by an influenza vaccination recommendation during the past pandemic season 2009-2010. Specifically, we constructed age controls interacted with indicator variables for the presence of a high-risk health condition as well as additional controls for regular contact with high-risk individuals, which mirrored the ACIP recommendation criteria for seasonal and pandemic vaccination of adults during the pandemic. In addition to detailed controls for adults' recommendation status for influenza vaccination during the 2009 influenza A/H1N1 pandemic, the model also included controls for respondents' sex, race, educational attainment, family income, and work status.

Statistical analyses were performed using STATA SE 10.1 software (StataCorp, College Station, TX, USA). Sample characteristics and unadjusted associations were computed using proportion estimation. Adjusted associations were computed using a probit model that incorporated all of the above controls simultaneously and were reported in terms of average partial effects, i.e., the average percentage point change in the probability of self-reported coverage by a government vaccination recommendation for influenza associated with a one-unit change in the value of a given variable of interest, controlling for other characteristics. All estimates were weighted to be nationally representative for the US adult population using probability weights that reflect the distribution of selected demographic characteristics of the US adult population as obtained from the US Current Population Survey (CPS). The RAND Corporation's Institutional Reviewed Board approved the study design and surveys.

\section{Results}

Overall, $46 \cdot 2$ percent of US adults correctly reported to be covered by a government recommendation for influenza vaccination during the 2010-2011 vaccination season (Table 1). Table 1 also reports unweighted sample sizes and weighted prevalence rates for all control variables along with their unadjusted associations with respondents' knowledge regarding their influenza vaccination recommendation status. Knowledge of influenza vaccination status varied significantly by race and ethnicity $(P=0.026)$, with the highest level of knowledge among non-Hispanic whites (49.1 percent). Likewise, knowledge was considerably higher among non-working than among working respondents $(67 \cdot 3$ percent versus 37.8 percent, $P<0.001)$ and among respondents who were recommended for seasonal vaccination (56.4 percent) or for both $\mathrm{H} 1 \mathrm{~N} 1$ and seasonal vaccination (54.8 percent) during the 2009-2010 pandemic $(P<0 \cdot 001)$. In addition, Table 1 shows considerable variation in self-reported coverage by a 2010-2011 government influenza vaccination recommendation across the different criteria of the targeted recommendations during the 2009-2010 pandemic. Adults aged 65 years and older with and without a high-risk health condition displayed the highest level of knowledge (88.3 percent and 84.4 percent), followed by adults aged 5064 years with a high-risk health condition ( 73.7 percent) and healthcare workers (67.5 percent). Self-reported coverage by a government vaccination recommendation was substantially lower among all other groups, rarely exceeding 50 percent.

Table 2 reports adjusted associations obtained from multivariable probit models that simultaneously control for socio-demographic characteristics and 2009-2010 recommendation status. The latter is measured using the more detailed set of controls for membership in a specific recommendation group to capture the large, age-related variation in self-reported coverage by a 2010-2011 government influenza vaccination recommendation within the different 2009-2010 recommendations groups document in Table 1. Accounting for 2009-2010 recommendation status, we found no statistically significant associations between knowledge of adults' current recommendation status and their socio-demographic characteristics with the exception of work status. Everything else equal, working adults were 9.6 percentage points less likely to know about being recommended for influenza vaccination than their nonworking counterparts.

In addition, the probit model showed that self-reported coverage by a government vaccination recommendation for the 2010-2011 vaccination season is closely related to age and health status that defined previous recommendations during the 2009-2010 pandemic and before. Persons aged 18-49 years with a high-risk health condition were $12 \cdot 9$ percentage points more likely to know about being recommended for influenza vaccination than adults who were not recommended for influenza vaccination in the past (omitted category). Persons age 50-64 without and with a high-risk health condition were 21 and $36 \cdot 7$ percentage points more likely to know about their current recommendation status than newly recommended persons who were not by an age- or health-related government influenza vaccination recommendation in the past. Corresponding average partial effects for persons age 65 and older without and with a high-risk health condition were 43.4 and $47 \cdot 8$ percentage points, respectively. Controlling for other factors, young healthy adults aged 18-24 years, who were only 
Table 1. Socio-demographic and health characteristics and their bivariate association with self-reported coverage by a government influenza vaccination recommendation, US adults, Fall 2010

\begin{tabular}{|c|c|c|c|}
\hline & \multirow[b]{2}{*}{ Unweighted $n$} & \multirow{2}{*}{$\begin{array}{l}\text { Weighted prevalence of } \\
\text { characteristic in } \%(95 \%-\mathrm{Cl})\end{array}$} & \multirow{2}{*}{$\begin{array}{l}\text { Self-reported coverage by government } \\
\text { vaccination recommendation } \\
\text { Weighted } \%(95 \%-\mathrm{Cl})\end{array}$} \\
\hline & & & \\
\hline Full sample & 2122 & 100 & $46 \cdot 2(43 \cdot 3 ; 49 \cdot 1)$ \\
\hline \multicolumn{4}{|l|}{ Sex } \\
\hline Male & 877 & $47 \cdot 7(44 \cdot 8 ; 50 \cdot 6)$ & $44 \cdot 6(40 \cdot 3 ; 49 \cdot 0)$ \\
\hline Female & 1245 & $52 \cdot 3(49 \cdot 4 ; 55 \cdot 2)$ & $47 \cdot 6(43 \cdot 8 ; 51 \cdot 4)$ \\
\hline \multicolumn{4}{|l|}{ Race and ethnicity } \\
\hline Non-hispanic white & 1841 & $74 \cdot 2(71 \cdot 3 ; 77 \cdot 2)$ & $49 \cdot 1(46 \cdot 1 ; 52 \cdot 2)$ \\
\hline Black & 133 & $10 \cdot 8(8 \cdot 7 ; 12 \cdot 9)$ & $37 \cdot 8(28 \cdot 0 ; 47 \cdot 7)$ \\
\hline Hispanic & 94 & $9 \cdot 7(7 \cdot 5 ; 11 \cdot 9)$ & $33 \cdot 8(22 \cdot 3 ; 45 \cdot 2)$ \\
\hline Other & 54 & $5 \cdot 3(3 \cdot 7 ; 6 \cdot 9)$ & $44 \cdot 6(29 \cdot 5 ; 59 \cdot 7)$ \\
\hline \multicolumn{4}{|l|}{ Level of education } \\
\hline High school or lower & 408 & $42 \cdot 3(39 \cdot 2 ; 45 \cdot 4)$ & $44.4(38.9 ; 49 \cdot 9)$ \\
\hline Some college & 808 & $27 \cdot 5(25 \cdot 3 ; 29 \cdot 7)$ & $46 \cdot 1(41 \cdot 9 ; 50 \cdot 2)$ \\
\hline College degree & 531 & $19 \cdot 7(17 \cdot 7 ; 21 \cdot 6)$ & $45 \cdot 3(40 \cdot 2 ; 50 \cdot 3)$ \\
\hline Masters degree or higher & 375 & $10 \cdot 5(9 \cdot 2 ; 11 \cdot 8)$ & $55 \cdot 4(49 \cdot 0 ; 61 \cdot 7)$ \\
\hline \multicolumn{4}{|l|}{ Family income } \\
\hline Less than $\$ 50,000$ & 913 & $49 \cdot 9(43 \cdot 9 ; 49 \cdot 8)$ & $47 \cdot 6(43 \cdot 1 ; 52 \cdot 0)$ \\
\hline$\$ 50,000$ to $\$ 74,999$ & 504 & $26 \cdot 0(23 \cdot 4 ; 28 \cdot 6)$ & $43 \cdot 6(38 \cdot 0 ; 49 \cdot 3)$ \\
\hline$\$ 75,000$ or more & 705 & $27 \cdot 1(24 \cdot 8 ; 29 \cdot 5)$ & $46 \cdot 2(41 \cdot 6 ; 50 \cdot 9)$ \\
\hline \multicolumn{4}{|l|}{ Currently working } \\
\hline No & 598 & $28 \cdot 4(25 \cdot 8 ; 31 \cdot 0)$ & $67 \cdot 3(62 \cdot 2 ; 72 \cdot 5)$ \\
\hline Yes & 1524 & $71 \cdot 6(69 \cdot 0 ; 74 \cdot 2)$ & $37 \cdot 8(34 \cdot 6 ; 41 \cdot 0)$ \\
\hline \multicolumn{4}{|c|}{ Overall recommendation status during the past influenza season } \\
\hline No vaccination recommendation & 387 & $24 \cdot 0(21 \cdot 4 ; 26 \cdot 7)$ & $22 \cdot 3(17 \cdot 1 ; 27 \cdot 6)$ \\
\hline H1N1 vaccination only & 49 & $4 \cdot 0(2 \cdot 6 ; 5 \cdot 4)$ & $18 \cdot 1(6 \cdot 2 ; 30 \cdot 1)$ \\
\hline Seasonal vaccination only & 962 & $38.8(36 \cdot 1 ; 41 \cdot 5)$ & $56 \cdot 4(52 \cdot 3 ; 60 \cdot 6)$ \\
\hline Both $\mathrm{H} 1 \mathrm{~N} 1$ and seasonal vaccination & 724 & $33 \cdot 2(30 \cdot 5 ; 36 \cdot 0)$ & $54 \cdot 8(49 \cdot 7 ; 60 \cdot 0)$ \\
\hline \multicolumn{4}{|c|}{ Detailed characteristics defining 2009-2010 recommendation status } \\
\hline Age $18-24$ years without high-risk condition & 96 & $6 \cdot 8(5 \cdot 1 ; 8 \cdot 4)$ & $23.9(14 \cdot 2 ; 33 \cdot 5)$ \\
\hline Age 50-64 years without high-risk condition & 583 & $16 \cdot 8(15 \cdot 1 ; 18 \cdot 6)$ & $51 \cdot 2(45 \cdot 9 ; 56 \cdot 5)$ \\
\hline Age $65+$ years without high-risk condition & 186 & $8 \cdot 1(6 \cdot 8 ; 9 \cdot 4)$ & $84 \cdot 4(78 \cdot 3 ; 90 \cdot 5)$ \\
\hline Age $65+$ years with high-risk condition & 151 & $9 \cdot 0(7 \cdot 2 ; 10 \cdot 7)$ & $88.3(82 \cdot 2 ; 94 \cdot 4)$ \\
\hline Household contact of high-risk person & 844 & $38.2(35 \cdot 5 ; 41 \cdot 0)$ & $52 \cdot 0(47 \cdot 5 ; 56 \cdot 5)$ \\
\hline Pregnant woman or contact of infant & 95 & $4 \cdot 8(3 \cdot 5 ; 6 \cdot 1)$ & $40 \cdot 2(27 \cdot 0 ; 53 \cdot 3)$ \\
\hline Age $18-49$ years with high-risk condition & 178 & $11 \cdot 8(9 \cdot 6 ; 13 \cdot 9)$ & $41 \cdot 4(31 \cdot 7 ; 51 \cdot 0)$ \\
\hline Age 50-64 years with high-risk condition & 245 & $7 \cdot 5(6 \cdot 3 ; 8 \cdot 7)$ & $73 \cdot 7(65 \cdot 5 ; 81 \cdot 9)$ \\
\hline Formal or informal healthcare worker & 286 & $12 \cdot 5(10 \cdot 7 ; 14 \cdot 4)$ & $67 \cdot 5(59 \cdot 9 ; 75 \cdot 1)$ \\
\hline
\end{tabular}

recommended for H1N1 vaccination during the 2009-2010 vaccination season, displayed a $4 \cdot 3$ percentage point lower levels of knowledge about being recommended for influenza vaccination during the 2010-2011 influenza season than healthy adults aged 25-49 years. Healthcare workers were 23.5 percentage points more likely to know about being recommended for influenza vaccination than newly recommended persons, but the corresponding association was considerably smaller and statistically insignificant for household contacts of high-risk individuals. Moreover, pregnant women or persons with regular contact with infants aged 6 months or less showed no increased knowl- edge about being recommended for influenza vaccination compared with newly recommended healthy adults aged $25-49$ years.

\section{Discussion}

Our study showed that at the start of the 2010-2011 influenza vaccination season, less than half of US adults knew that they were recommended for influenza vaccination by the US government. This finding highlights the magnitude of outreach required of public health officials and healthcare providers to increase adults' awareness of their 
Table 2. Multivariable probit model for self-reported coverage by a government influenza vaccination recommendation with socio-demographic and health controls, US adults, Fall 2010

\section{Average partial effect}

Estimate in percentage points $(95 \%-\mathrm{Cl})$

\begin{tabular}{|c|c|}
\hline \multicolumn{2}{|l|}{ Sex } \\
\hline Male & Reference category \\
\hline Female & $1 \cdot 5(-3 \cdot 4 ; 6 \cdot 5)$ \\
\hline \multicolumn{2}{|l|}{ Race and ethnicity } \\
\hline Non-hispanic white & Reference category \\
\hline Black & $-3 \cdot 5(-12 \cdot 7 ; 5 \cdot 6)$ \\
\hline Hispanic & $-5 \cdot 4(-16 \cdot 2 ; 5 \cdot 4)$ \\
\hline Other & $0 \cdot 5(-12 \cdot 7 ; 13 \cdot 7)$ \\
\hline \multicolumn{2}{|l|}{ Level of education } \\
\hline High school or lower & Reference category \\
\hline Some college & $2 \cdot 4(-3 \cdot 6 ; 8 \cdot 4)$ \\
\hline College degree & $1 \cdot 9(-4 \cdot 7 ; 8 \cdot 5)$ \\
\hline Masters degree or higher & $7 \cdot 3(-0 \cdot 5 ; 15 \cdot 1)$ \\
\hline \multicolumn{2}{|l|}{ Family income } \\
\hline Less than $\$ 50,000$ & Reference category \\
\hline$\$ 50,000$ to $\$ 74,999$ & $-0 \cdot 4(-6 \cdot 8 ; 6 \cdot 0)$ \\
\hline$\$ 75,000$ or more & $-0 \cdot 1(-6 \cdot 1 ; 6 \cdot 0)$ \\
\hline \multicolumn{2}{|l|}{ Currently working } \\
\hline No & Reference category \\
\hline Yes & $-9 \cdot 6(-16 \cdot 7 ;-2 \cdot 4)$ \\
\hline \multicolumn{2}{|c|}{ Detailed recommendation status during the past influenza season } \\
\hline No vaccination recommendation & Reference category \\
\hline \multicolumn{2}{|l|}{ H1N1 vaccination only } \\
\hline Age 18-24 without high-risk condition & $-4 \cdot 3(-14 \cdot 7 ; 6 \cdot 2)$ \\
\hline \multicolumn{2}{|l|}{ Seasonal vaccination only } \\
\hline Age 50-64 without high-risk condition & $21 \cdot 0(15 \cdot 1 ; 26 \cdot 8)$ \\
\hline Age 65 + without high-risk condition & $43 \cdot 4(37 \cdot 3 ; 49 \cdot 5)$ \\
\hline Age $65+$ with high-risk condition & $47 \cdot 8(41 \cdot 9 ; 53 \cdot 6)$ \\
\hline Household contact of high-risk person & $4 \cdot 9(-0 \cdot 4 ; 10 \cdot 1)$ \\
\hline \multicolumn{2}{|l|}{ Both H1N1 and seasonal vaccination } \\
\hline Pregnant woman or contact of infant & $0.0(-11 \cdot 5 ; 11 \cdot 5)$ \\
\hline Age $18-49$ years with high-risk condition & $12 \cdot 9(4 \cdot 2 ; 21 \cdot 5)$ \\
\hline Age 50-64 years with high-risk condition & $36 \cdot 7(30 \cdot 0 ; 43 \cdot 4)$ \\
\hline Formal or informal healthcare worker & $23 \cdot 5(15 \cdot 7 ; 31 \cdot 2)$ \\
\hline
\end{tabular}

recommendation status. Awareness of one's recommendation status was especially low among young healthy adults who were not recommended for any type of influenza vaccination or recommended for H1N1 vaccination only during the 2009-2010 pandemic vaccination season. The latter finding is especially noteworthy as the inclusion of the 2009 influenza A/H1N1 strain in the 2010-2011 influenza vaccine was aimed at protecting this highly vulnerable population group in particular. ${ }^{7,8}$ The relatively low levels of awareness among newly recommended adults compared to adults who have already been recommended for seasonal influenza vaccination in the past also reveal limited awareness of last year's changes in ACIP's influenza vaccination recommendations and highlight the need for additional communication efforts highlighting the universal nature of the new vaccination recommendations for influenza. Particularly, as awareness seems especially low among working adults, employer-based outreach programs such as employer-based reminder systems and company flu shot clinics may be a promising tool for increasing awareness and cost-effective vaccine use among newly targeted young, healthy adults. ${ }^{9}$ Similarly, the comparatively low rates of awareness among certain traditional recommendation groups such as pregnant women and household contacts of infants call for increased efforts to highlight the importance of influenza vaccination for pregnant women and caregivers of young children during routine care visits to obstetricians or pediatricians.

Our study has several limitations. First, despite adopting a probability-based sample design that covers both online 
and offline households, the decision to participate in the ALP could be associated with awareness of one's recommendation status in a way that compromises the external validity of our findings. Likewise, as many ALP members participated in several influenza vaccination surveys during the pandemic, "learning effects" or panel conditioning may have affected our results. Particularly, our estimates of knowledge of adults' recommendation status are somewhat larger compared with previous estimates of adults' awareness of their influenza vaccination recommendation status during the pandemic ${ }^{6}$. This difference may reflect genuine increases in the awareness of adults with regard to government influenza vaccination recommendations or confusion during the pandemic with regard to the distinct recommendations for seasonal and pandemic vaccination, respectively, but may also reflect differences in sample source, survey methodology, or potential learning effects. In general, our estimates appear conservative, as both potential biases mentioned earlier suggest that adults' awareness of their government recommendation status for influenza vaccination is, if anything, estimated too high.

The new universal vaccination recommendation for influenza should facilitate the streamlining of public health communication and the initiation of counseling by healthcare providers about the benefits of annual influenza vaccination. This is because compliance with official recommendations no longer requires time-consuming administrative procedures aimed at identifying subgroups of recommended patients based on their age, health, or contact with high-risk individuals, many of which are not routinely documented in medical record and other administrative data. In this way, messaging can more efficiently address the attitudinal and access barriers to vaccination irrespective of demographic and clinical characteristics. Eliminating the administrative complexity associated with targeting recommended subgroups based on demographic and clinical characteristics should facilitate the implementation of evidence-based, but underused strategies for communicating the benefits of vaccination, including oneon-one counseling by healthcare providers during office visits, the use of phone, email, or postcard patient reminders of physicians and employers or the increased use of social media. ${ }^{10-19}$ However, the universal vaccination recommendations will only be successful if they reach both patients and physicians and lead to changing vaccination practices. It has never been easier.

\section{Acknowledgements}

Financial support for the data collection underlying this study was provided by the National Institute on Aging (NIA) under grant R01AG20717 (PI: Kapteyn).

\section{Conflict of interest}

The authors have no conflicts of interest to declare and have no financial disclosures.

\section{References}

1 Centers for Disease Control and Prevention. Prevention and control of influenza with vaccines. Recommendations of the Advisory Committee on Immunization Practices (ACIP), 2010. MMWR Recomm. Rep. Early Release 2010; 59:1-62.

2 Centers for Disease Control and Prevention. Recommendations and guidelines: Advisory Committee on Immunization Practices (ACIP). Available at http://www.cdc.gov/vaccines/recs/acip/ (Accessed 6 December, 2010)

3 Centers for Disease Control and Prevention. CDC's Advisory Committee on Immunization Practices (ACIP) recommends universal annual influenza vaccination. Press release: 24 February, 2010. Available at http://www.cdc.gov/media/pressrel/2010/r100224.htm (Accessed 9 August, 2011).

4 Centers for Disease Control and Prevention. Prevention and control of influenza with vaccines: Recommendations of the Advisory Committee on Immunization Practices (ACIP), 2009. MMWR Recomm Rep 2009; 58:1-52.

5 Davis M, McMahon S, Santoli J, Schwartz B, Clark S. A national survey of physician practices regarding influenza vaccine. J Gen Intern Med 2002; 17:670-676.

6 Maurer J, Uscher-Pines L, Harris K. Awareness of government seasonal and 2009 H1N1 influenza vaccination recommendations among targeted U.S. adults: the role of provider interactions. Am J Infect Control 2010; 38:489-490.

7 Centers for Disease Control and Prevention. Use of influenza A (H1N1) 2009 monovalent vaccine: Recommendations of the Advisory Committee o Immunization Practices (ACIP), 2009. MMWR Early Release 2009; 58:1-8.

8 World Health Organization. Recommended viruses for influenza vaccines for use in the 2010-2011 northern hemisphere influenza season. (18 February 2010, revised 5 March 2010, cited 6 December 2010). Available at http://www.who.int/entity/csr/disease/influenza/ 201002_Recommendation.pdf (Accessed 06 December 2010).

9 Nichol KL, Lind A, Margolis KL et al. The effectiveness of vaccination against Influenza in healthy, working adults. N Engl J Med 1995; 333:889-893.

10 Szilagyi P, Bordley C, Vann JC et al. Effect of patient reminder/ recall interventions on immunization rates: a review. JAMA 2000; 284:1820-1827.

11 Jacobson Vann JC, Szilagyi P. Patient reminder and recall systems to improve immunization rates. Cochrane Database Syst Rev 2008; 1:1-71.

12 Poland G, Shefer A, McCauley M, Webster P, Whitley-Williams P, Peter G. Standards for adult immunization practices. Am J Prev Med 2003; 25:144-150.

13 Fukuda K, Bridges C, Brammer TL, Izurieta HS, Cox N. Prevention and control of influenza: Recommendations of the Advisory Committee on Immunization Practices (ACIP). MMWR Recomm Rep 1999; 48:1-30.

14 Johnson DR, Nichol KL, Lipczynski K. Barriers to adult immunization. Am J Med 2008; 121:S28-S35.

15 Maurer J, Harris K. The scope and targeting of influenza vaccination reminders among U.S. adults: evidence from a nationally representative survey. Arch Intern Med 2010; 170:390-392. 
Maurer et al.

16 Maurer J, Harris K, Lurie N. Reducing missed opportunities to vaccinate adults against influenza: what's realistic? Arch Intern Med 2009; 169:1633-1634.

17 Ahluwalia IB, Jamieson DJ, Rasmussen SA et al. Correlates of seasonal influenza vaccine coverage among pregnant women in Georgia and Rhode Island. Obstet Gynecol 2010; 116:949955.
18 Van Buynder PG, Carcione D, Rettura $V$ et al. Marketing pediatric influenza vaccination: results from a major metropolitan trial. Influenza Other Respi Viruses 2010; 5:33-38.

19 Centers for Disease Control and Prevention. National influenzavaccination week to focus on young adults: CDC uses social media to spread message that even healthy young adults can get the flu. Press release: 10 December 2010 (Accessed 9 August, 2011). 